\title{
On Lexical Borrowing from English into Chinese via Transliteration
}

\author{
Yan Chen ${ }^{1}$ \\ ${ }^{1}$ Yancheng Teachers University, China \\ Correspondence: Yan Chen, Department of English Education, School of Foreign Languages, Yancheng Teachers \\ University, Yancheng 224002, Jiangsu, China. E-mail: cyan@xmu.edu.cn
}

$\begin{array}{ll}\text { Received: July 30, } 2013 & \text { Accepted: August 16, } 2013 \quad \text { Online Published: September 26, } 2013 \\ \text { doi:10.5539/ells.v3n4p1 } & \text { URL: http://dx.doi.org/10.5539/ells.v3n4p1 }\end{array}$

Foundation item: a research project funded by Yancheng Teachers University (license number: 12YSYJB0105)

\begin{abstract}
Transliteration has played an important role in lexical borrowing from foreign languages into Chinese. In this paper the question of lexical borrowing from English into Chinese via transliteration is treated from multiple perspectives with data drawn from A Dictionary of Loan Words and Hybrid Words in Chinese, the most authoritative dictionary of loanwords in Chinese so far. It is found that as a method of adaptation, transliteration is used in three ways, namely phonetic transcription, transliteration plus notes, and half transliteration plus half translation, which bring into being three subtypes of transliterations respectively: phonemic loans, annotated transliterations, and loanblends. Three strategies have been adopted to add semantic transparency to transliterations: direct labeling of semantic category with radicals or characters, indirect suggestion of meaning by combining characters in syntagmatic lexical relations conforming to Chinese word-formation processes, and addition of meaning through endowing transliterations with positive, negative, or jocular connotations. An important means to enrich the Chinese lexicon and promote products in advertising language as it is, transliteration poses problems of understanding, including distortion of meaning and folk-etymological interpretation.
\end{abstract}

Keywords: English, Chinese, transliteration, subtypes, semanticization, merits, demerits

\section{Introduction}

When different languages come into contact, the languages involved may be affected in various ways. "The simplest kind of influence that one language may exert on another is the "borrowing' of words" (Sapir, 1921). Borrowing takes place when speakers of one language reproduce a pattern previously found in another (Haugen, 1950). The term "borrowing" can be used to describe both the end product and the process itself, as in Cannon (1988) and Moody (1996), but in this paper, it is restricted to the process. During the borrowing process, the "borrower" language becomes "recipient" language while the "source" language becomes "donor" language. When a recipient culture borrows a new item or concept, its language may borrow the name of that item or concept from the donor language, thus adding to the recipient lexicon a "loanword". Loanwords specifically refer to words borrowed from one language into another, which have become lexicalized, i.e., assimilated phonetically, graphemically, and grammatically, into the new language (Bussmann, 1996).

In the history of the Chinese language, there have been three periods of intensive borrowing. In the first period, dating from Western Han (206 BC-AD 24) to the Song Dynasty (960-1279), Chinese borrowed extensively words and expressions from such Central Asian languages as Persian and Arabic as well as Buddhist terms from Sanskrit resulting respectively from contact with ancient tribes along the Silk Road and translation of Buddhist scriptures. The second period started when the Jesuit missionaries, together with the Dominicans and Franciscans, in the late Ming (AD 1368-1644) and early Qing (AD 1616-1911) dynasties, started to introduce into China Christianity and Western learning. Translation of works on a wide variety of subjects in both natural and social sciences gave birth to numerous neologisms, many of which are loanwords in common use today. This wave of translating and diffusing Western learning extended well into the middle of the $20^{\text {th }}$ century when close relations with the former USSR brought into Chinese a number of loanwords from Russian. The third period began with the implementation of reform and opening-up in the early 1980s. As China resumed normal relations with a large number of foreign countries, heavy borrowing from foreign languages has become an irresistible 
trend. In each of these periods, lexical borrowing from different languages, for instance, Sanskrit, Latin, English, Russian, differs in terms of time span, volume and influence.

In the analysis of loanwords in Chinese, loanwords are usually classified according to the method of adaptation, i.e., whether adaptation is done according to pronunciation or meaning, and opinions remain divided over what types of new formations, either inspired by or modeled on foreign words, should be considered as loanwords. One group of scholars, including Lü (1942), Sun (1957), Wang (1958), Gao and Liu (1958), and Shi (1997), confine loanwords to transliterations and graphic loans from Japanese, excluding semantic loans on the ground that such loans consist solely of native morphemes. Another group of scholars, including Luo (1950), Chan and Kwok (1990), Masini (1993) and Huang (1995), include in the category of loanwords both loan translations and semantic loans. Nevertheless, one consensus has been reached, i.e., transliterations are loanwords in Chinese. As "the action, process, or result of converting one set of signs to another" (McArthur, 1998), transliteration functions as a basic strategy of lexical borrowing in Chinese, just as transliterations constitute the most typical of loanwords. Apart from being a useful method of adaptation, transliteration has important consequences for lexicological studies.

\section{Methods of Transliteration and Subtypes of Transliterations}

Before any classification of transliterated loanwords is offered, priority needs to be given to clarification of some terms, as terminologies of borrowing differ among scholars. Different scholars may use different terms for the same borrowing process or end product. For instance, in Masini's (1993) classification of loanwords in Chinese, he uses the terms "phonemic loans," "loan translations" and "semantic loans" the same way as Haugen (1950) does, but uses the term "hybrid" in a different sense. According to him, a hybrid is composed of a phonemic loan plus an autochthonous element, usually used to indicate the semantic category of the phonemic loan, as in the case of 坦克车 (<tank). In Haugen's (1950) discussion, however, the term "hybrid" designates "loanwords in which only a part of the phonemic shape of the word has been imported, while a native portion has been substituted for the rest". For our purpose of research, we would adopt some of Haugen's terms and approaches. First, to name a borrowing process, the pattern in the donor language that is borrowed is called the model, and the attempted reproduction in the recipient language is called the replica. Secondly, we would categorize loanwords through analyzing what the borrower does by comparing the pattern that he is reproducing with the results that he succeeds in turning out, i.e., comparing the replica with its model, phonetically before semantically.

In order to avoid any subjective and random classification of transliterations, we have created a corpus of loanwords from English by keying in such words included in A Dictionary of Loan Words and Hybrid Words in Chinese (Liu, Gao, Mai, \& Shi, 1984), the most exhaustive of its type in China so far. The dictionary contains around 10000 loanwords which are phonetically associated with their models, or 7704 nonduplicated loanwords when the variant forms are deleted (Shi, 2000). It should be noted that our corpus only includes loanwords directly from English, with loanwords borrowed via transmission languages such as Japanese excluded, simply to facilitate our classification by evading the intricate issue of lexical borrowing between Chinese and Japanese. The corpus contains 4645 loanwords from English in total, but the total number is reduced to 3397 if we delete the 1248 variant forms. This corpus is deemed intact for all the loanwords in the dictionary are phonetically connected to their models in one way or another, i.e., they are all transliterations, as is stipulated by the principles of entry selection of the dictionary.

After tabulating and categorizing each loanword according to correspondence with its respective English model, we find that transliteration is a broad term covering three different ways of adaptation and consequently three subtypes of transliterations, which will be exemplified in more detail in the following sections.

\subsection{Phonetic Transcription and Phonemic Loans}

When a lexical item has no ready equivalent in the recipient language, the easiest way to adapt it is to transcribe it phonetically. By phonetic transcription, the phonetic patterns of the model are reproduced with Chinese characters to simulate the original pronunciation, though the degree of phonetic resemblance to the model may vary from replica to replica. Loanwords thus created are called phonemic loans, and they amount to 3055 , making up $65.8 \%$ of all entries in our corpus. Transliterations like 巴士 $(<$ bus $)$, 罗曼蒂克 $(<$ romantic), 图腾 $(<$ totem), 柠檬 $(<$ lemon), 仙客来 $(<$ cyclamen) and 幽浮 $(<\mathrm{UFO})$ all belong to this category. In phonemic loans, the Chinese characters serve an essential function of representing a pronunciation similar to that of the replica. 


\subsection{Transliteration plus Notes and Annotated Transliterations}

Sometimes, the coiner of a transliteration makes a special effort to crystallize part of the meaning of the model through integrating a note into the replica. By transliteration with notes, a Chinese morpheme is added to a phonetic transcription, in a way to supply additional information, usually about the semantic category of the model, thus creating what may be called annotated transliterations. The total number of transliterations thus created is 541 , making up $11.6 \%$ of all entries in our corpus. What is more, The position of the note may vary, either at the end of a replica, as in 卡车 $(<\mathrm{car})$, 嘉年华会 (<carnival), 仑巴舞 (<rumba), 华夫饼干 $(<$ waffle) and 恤衫 $(<$ shirt), or in rare cases, at the beginning of a replica, as in 酒吧 $(<$ bar $)$ and 车胎 $(<$ tire/tyre).

\subsection{Half Transliteration plus Half Translation and Loanblends}

As a model may comprise several morphemes, a coiner of transliterations may adapt the morphemes either by phonetic transcription or by semantic translation. By joint use of transliteration and semantic translation, one part of the model is transliterated while the rest is a morpheme-for-morpheme rendition, thus creating a loanblend, the total number of which reaches 1049 , or $22.6 \%$ in our corpus. Examples of this type include 千瓦 ( $<$ kilowatt), 奶昔 (<milkshake), 登革热 (<dengue fever), 霓虹灯 (<neon lamp), 拓扑学 (<topology), etc.

\section{Semanticization of Transliterations}

As is shown above, Chinese transliterations of English are replicas that have partial or complete phonemic similarity to their models. The phonetic adaptations basically serve a phonographic function, which is why the same model has several distinct phonemic loans, as in the case of 华尼拉 (<vanilla) whose variant transliterations amount to eleven, and 巧克力 (<chocolate) which has seven variant phonemic loans. This also accounts for the fact that some transliterations at the time of creation were unintelligible to native speakers of Chinese, like 德律风 (<telephone), 赛因斯 ( $<$ science), 德谟克拉西 (<democracy), 烟士披利纯 (<inspiration) and 巴斯克拉里涅特 (<bass clarinet).

However, due to the pleremic nature of the Chinese script and the well-known tendency of Chinese to resist lexical borrowing through phonemic transliteration (Chan et al., 1990; Masini 1993; Chen, 1999), transliterations are prone to semanticization, i.e., the process of linking the referent or sense of a replica to its model to make transparent the meaning of originally opaque loanwords. In order that transliterations embody semantic connection of some sort to their models, three strategies are found to be at work.

\subsection{Direct Labeling of Semantic Category}

One conventional option to hint at some semantic value of phonemic loans is to employ relevant radicals (Pan, 1957). For instance, in transliteration of plant names, the radicals 木 and \# are used, as in 柠檬 (<lemon), 柿苓 (<stillingia) and 蔻蔻 $(<$ cocoa). As the radicals are ideographic, they are suggestive of one semantic component of the replicas. Similarly, replicas with the radical $\square$ indicate that they designate something edible or drinkable, as in 咖啡 (<coffee) and 咖喱 (<curry).

A second option is to select words which are both phonetically and semantically connected to the model. For instance, 声呐 (<sonar) suggests that the referent of the replica has something to do with sound by the morpheme 声 (sound), and 绷带 (<bandage) indicates the shape of the referent with the word 带 (strap).

A third option is to create an annotated transliteration. The morpheme 鸟, for instance, is put at the end of transliterations of bird names to mark the semantic category of the replicas, as in 克沙尔鸟 (<quetzal/quezal), 茄卡那鸟 (<jacana), 几维鸟 (<kiwi), 渡渡鸟 (< dodo), and 维丽俄鸟 $(<$ vireo).

In the above examples, a single radical or character acts as a key to the semantic category of replica. In other words, proper radicals and words are signposts indicating part of the meaning of replicas; ingenious use of them may give full play to their ideographic value and increase the intelligibility of transliterations. However, as phonetic reproductions, characters in transliterations do not guarantee invariably correct suggestion of meaning. The replica 水门汀 (<cement), for instance, has nothing to do with either 水 (water) or 汀 (spit of land).

\subsection{Indirect Suggestion of Meaning}

In addition to direct labeling, the meaning of a replica can be suggested in a roundabout way when the replica in question is taken into account as a whole. The combination of characters, whose syntagmatic lexical relations conform to Chinese word formation-processes, even if in a loose way, may become more or less analyzable and give multiple hints at the meaning of the replica. For example, 幽默 consists of 幽 (secluded, deep and remote) and 默 (silent, quiet). "Semantically, the choice of characters might reflect that being shrewd and reserved is not incompatible with a sense of humour; some Chinese think that a humorous person should maintain a 
dignified silence while others laugh at his/her jokes" (Zuckermann, 2003). Transliterations like 爱斯不难读 $(<$ Esperanto), 引得 (<index), 舍德主义 $(<$ sadism), 踢踏舞 $(<$ tittup), and 霓虹 $(<$ neon) all leave more than one clue for inference of meaning. In these transliterations, the selection of characters renders the transliterations analyzable by containing more than one clue to the semantic components of the referents, and involves less effort of interpretation than purely phonemic loans and more than semantic loans when users try to approach the meaning of them.

\subsection{Addition of Meaning}

Connotation includes the associative, emotive, social, or stylistic meaning of words. In transliterations, characters may build into the meaning of a replica some connotations originally missing in its model. The connotative potential is particularly manifest in the associative meaning of replicas. Due to the inherent meanings of the characters, some transliterations may arouse positive associations. For instance, 甘蜜 (<gambier/gambir) and 可口可乐 (<coca cola) literally mean sweet honey and delicious and enjoyable respectively, which lend to their positive associations among Chinese users. The same sort of positive associations may be motivated in some other transliterations, too, like 密实大衣 (<maxicoat), 香波 ( $<$ shampoo), and 赐保命 (<spermine).

In contrast to appreciative connotations, the word “infidel", for instance, is transliterated into 婴匪毒 (baby-bandit-poison), the latter two characters of which connect the referent to something evil, making the original meaning derogatory and negative. The transliteration 拖尸 (<toss), instead of exhibiting the fun and excitement of the practical joke of tossing a new student into a lake, establishes a link between the activity and a corpse. This is because certain characters in transliterations may evoke negative imagery and associations, which can also be seen in 发拉屎 (<fallacy), 大笨钟 $(<$ Big Ben), 拖肥 $(<$ toffee/toffy), 印发热凶 (<inflation), 协 识脱离 $(<$ hysteria $)$ and 滥斧 $(<\mathrm{lamp})$.

In addition to emotive meanings, a few transliterations are jocular adaptations. The replica 黑漆板登 $(<$ husband) depicts the husband as a black-painted bench, 尖头鳗 (<gentleman) portrays the gentleman as an eel with a pointed head, 弹性女郎 (<dancing girl) stresses the elasticity of such a girl, 迷你裙 (<miniskirt) conceals the size of the skirt but gives prominence to the purpose of the skirt- to enchant you, and 糖果舞 (<tango) links the dance to candies. Such substitution of images contributes nothing to crystallization of meaning but sends speakers of Chinese laughing.

\section{Transliteration as a Two-Edged Sword}

In present-day China, lexical borrowing from English is an on-going process. The same methods of transliteration are used more frequently than ever and new transliterations are introduced at an increasing speed. A survey of neologisms has revealed that loanwords from English alone in 1992 and 1993 made up respectively $80.95 \%$ and $82.14 \%$ of all the loanwords found in those two years (Sun \& Jiang, 2000) as against a total of 27 loanwords from foreign languages, a mere $0.75 \%$ among the 3600 neologisms that emerged during the 1949-1978 period, and as against 58 loanwords, or 3.4\% among the 1700 neologisms in the 1978-1988 period (Wang, 1993). As a means to enrich the Chinese vocabulary, transliteration is of great help to fill lexical voids speedily, although the efficacy of transliterations awaits the test of time.

At the same time, transliteration may pose various problems of understanding for Chinese users in that purely phonemic loans are incomprehensible and the characters in some semanticized transliterations may affect understanding of their true meanings. In some cases, misunderstanding may arise if the characters motivate negative semantic links to the referents. As is mentioned above, semanticized transliterations can only be suggestive of rather than convey the precise meaning of loanwords, so it is a tricky business to integrate semantic components into transliterations, The replica 来福枪 $(<$ rifle), for instance, links the weapon to blessing, which is rather unacceptable to some people who, in turn, would prefer its neutral replica 来复枪. The replica 爱滋病 ( $<$ AIDS), allows for a connection of love to the generation of the disease, an undesirable association which may accounts for the greater popularity of its more neutral variant 艾滋病. A related type of misunderstanding is folk-etymological interpretation of transliterations. The replica 大丽花 $(<$ dalia), is mistaken for a type of flower native to Dali, an autonomous prefecture in Yunnan Province, just as 的确良 ( $<$ Dacron) is assumed be so named for good qualities (Zhang, 2000).

Problematic as it is, transliteration has proved to be an effective means in advertising, especially when pleasant imagery or association is needed. A case in point is the replica of the word "coca cola". In 1927, a beverage with the name 蝌蝌啃蜡 (tadpole-tadpole-bite-wax) appeared in Shanghai. With such a weird name and weird taste, the beverage was unpopular among the local residents. In the next year, the Coca-Cola company sponsored a naming contest for the product, in which Professor Jiang Yi, a man from Shanghai living in London at the time, 
won a $£ 350$ prize with the coinage 可口可乐. This semanticized coinage, with its high intelligibility and positive associative meanings, has in part contributed to the success of the product on Chinese market (Tang, 2008). Nowadays, it is a common practice in the Chinese advertising circle to exploit characters rich in positive associations in the transliteration of brand names. The two brand names 舒肤佳 (to comfort-skin-good) ( $<$ Safeguard, a brand name of soap) and 悍马 (brave-horse) ( $<$ Hummer, a brand name of an automobile), for example, "arouse an association of the soap product 'Safeguard' with ideal skincare functions and the car 'Hummer' with speed and strength" (Miao, 2005).

\section{Conclusion}

Transliteration has been used in lexical borrowing from foreign languages into Chinese for a long time and the issues surrounding it have attracted the attention of many researchers. A search for titles containing the words "loanwords" or "borrowing" in CNKI (China National Knowledge Infrastructure) will yield about 1500 results dating from 1992. Unlike most researches in which authors rely on subjective observation of examples, this study has offered a more thorough examination of transliteration by using data garnered from the most exhaustive dictionary of loanwords in Chinese. It is found that transliteration is used in three ways, i.e., phonetic transcription, transliteration plus notes, and half transliteration plus half translation, which bring into being three subtypes of transliterations respectively: phonemic loans, annotated transliterations, and loanblends. As Chinese people tend to resist lexical borrowing through phonemic transliteration, three strategies have been adopted to add semantic transparency to transliterations: direct labeling of semantic category with radicals or characters, indirect suggestion of meaning by combining characters in syntagmatic lexical relations conforming to Chinese word-formation processes, and addition of meaning through endowing transliterations with positive, negative, or jocular connotations. An important means to enrich the Chinese lexicon as it is, transliteration poses problems of understanding, including distortion of meaning and folk-etymological interpretation.

The findings of this paper bear on several important issues of transliteration. First, the classification of transliteration and transliterations is made by examining how a replica corresponds to its model in two steps: compare the pronunciation of a replica with its model to see if it is a partial or complete phonetic transcription, and if the replica contains elements not phonetically corresponding to the model, identify where those elements are from. This leaves out of consideration an often-quoted type of transliteration, 音兼义 (sound-concurrent with-meaning) (Luo, 1950) or 音义兼顾 (sound-meaning-both-to consider) (Gao et al., 1958), or 谐音音译 (pun-sound-transliteration) (Shi, 2000). These three terms stress the somewhat close correlation between a phonemic loan and its model in both meaning and pronunciation, so they fall into the scope of semanticization, or into a subtype of phonemic loans which differs from purely phonemic loans whose pronunciations motivate no links to meaning. Second, it is found that efforts are made by coiners of transliterations to semanticize their coinages. One norm established in lexical borrowing in Chinese is when there are alternatives, transliterations give way to words formed by the other methods, preferably by the method of semantic translation (Chen, 1999). The analysis of semanticization strategies has proved that semantic transparency has been an important concern in transliteration. Third, the exploration of connotations of transliterations suggests that coiners of transliterations need to be keenly aware of possible associations of characters. In China, language planning institutions like the official Xinhua News Agency has, since the 1950s, compiled handbooks of foreign sounds and Chinese characters to be used in the transliteration of people's names, and in 1993, published the dictionary Names of the World's Peoples-A Comprehensive Dictionary Names in Roman-Chinese, with a coverage of 650000 entries concerning 55 languages used in over 100 countries and regions. However, the authority of the agency is constantly challenged as mass media like television and the Internet spread deviant transliterations at a speed too rapid for the agency to correct or check them. With a substantial number of homophonous characters at hand, coiners need to be on alert for unintended associations in an effort to bring into full play the expressive power of characters.

This study has not touched upon some other issues of transliteration, for instance, the use of Chinese dialects in transliteration, and differences in transliterations particular to specific areas like Hong Kong, Macau and Taiwan, the problem of variant forms of the same model, etc. A probe into these questions will yield equally interesting results.

\section{References}

Bussmann, H. (1996). Routledge dictionary of language and linguistics (G. P. Trauth, \& K. Kazzazi, Trans. \& Ed., p. 287). London: Routledge.

Cannon, G. (1988). Chinese borrowings in English. American Speech, 63(1), 3-33.
http://dx.doi.org/10.2307/455420 
Chan, M., \& Kwok, H. (1990). A study of lexical borrowing from English in Hong Kong Chinese. Hong Kong: University of Hong Kong Center of Asian Study.

Chen, P. (1999). Modern Chinese: History and sociolinguistics (pp. 99-112). Cambridge: Cambridge University Press. http://dx.doi.org/10.1017/CBO9781139164375

Gao, M., \& Liu, Z. (1958). A study of loanwords in modern Chinese (pp. 8, 163-165). Beijing: Script Reform Press.

Haugen, E. (1950). The analysis of linguistic borrowing. Language, 26(2), 210-231. http://dx.doi.org/10.2307/410058

Huang, H. (1995). Chinese loanwords born under foreign influence. Lexicon Construction Newsletter, 7, 14-26.

Liu, Z., Gao, M., Mai, Y., \& Shi, Y. (1984). A dictionary of loan words and hybrid words in Chinese. Shanghai: Shanghai Lexicographical Publishing House.

Luo, C. (1950). Language and culture (pp. 18, 28). Beijing: Beijing University Press.

Lü, S. (1942). An outline of Chinese grammar (p. 19). Beijing: Commercial Press.

Masini, F. (1993). The formation of modern Chinese lexicon and its evolution towards a national language: The period from 1840 to 1898. Journal of Chinese Linguistics, Monograph Series, (1993)6.

McArthur, T. (1998). The Oxford concise companion to the English language (p. 618). Oxford: Oxford University Press.

Miao, R. (2005). Loanword adaptation in Mandarin Chinese: Perceptual, phonological and sociolinguistic factors (Doctoral dissertation, p. 31). Stony Brook: State University of New York. Retrieved from https://linguistics.stonybrook.edu/sites/default/files/uploads/Miao_2005.pdf

Moody, A. J. (1996). Transmission languages and source languages of Chinese borrowings in English. American Speech, 71(4), 405-420. http://dx.doi.org/10.2307/455714

Pan, Y. (1957). Loanwords in Chinese before the Opium War. Journal of Sun Yat-sen University, 3, 98-113.

Sapir, E. (1921). Language (p. 206). New York: Harcourt, Brace and Company.

Shi, Y. (1997). Revisiting terminologies of Chinese loanwords. Lexicon Construction Newsletter, 12, 17-19.

Shi, Y. (2000). Chinese loanwords (pp. 162-163, 127-128). Beijing: Commercial Press.

Sun, C. (1957). Chinese Lexicon (p. 316). Changchun: Jilin People's Press.

Sun, H., \& Jiang, K. (2000). A study of recent borrowings in Mandarin. American Speech, 75(1), 98-106. http://dx.doi.org/10.1215/00031283-75-1-98

Tang, Z. (2008, December 10). Deciphering cola products: Coca Cola originally named Kekekenla. Beijing Daily (p. 4). Retrieved from http://society.people.com.cn/GB/8491939.html

Wang, L. (1958). Manuscript on Chinese history (Volume II, p. 526). Beijing: Science Press.

Wang, T. (1993). New loanwords in Chinese: A cultural psychological perspective. Chinese Language Learning, $1,35-40$.

Zhang, S. (2000). A study of Chinese folk etymology (pp. 145-147). Beijing: Language \& Culture Press.

Zuckermann, G. (2003). Language contact and lexical enrichment in Israeli Hebrew (p. 60). New York: Palgrave Macmillan. http://dx.doi.org/10.1057/9781403938695

\section{Copyrights}

Copyright for this article is retained by the author(s), with first publication rights granted to the journal.

This is an open-access article distributed under the terms and conditions of the Creative Commons Attribution license (http://creativecommons.org/licenses/by/3.0/). 